\title{
Comparação de perfis de dissolução da cefalexina através de estudos de cinética e eficiência de dissolução (ED\%)
}

\author{
Cristina Helena dos Reis Serra*, Sílvia Storpirtis
}

Departamento de Farmácia, Faculdade de Ciências Farmacêuticas, Universidade de São Paulo

*Correspondência:

C. H. .R. Serra

Departamento de Farmácia

Laboratório de Farmacotécnica

Universidade de São Paulo

Av. Prof. Lineu Prestes, 580,

Cidade Universitária,

05508-000 - São Paulo - SP, Brasil

Email: chserra@usp.br
O presente estudo reporta os resultados comparativos obtidos através da avaliação da cinética e da eficiência de dissolução da cefalexina a partir de dois lotes ( 1 e 2) de diferentes produtos contendo tal fármaco disponíveis no mercado brasileiro (A e B) sob a forma de comprimidos de liberação convencional. Os perfis de dissolução foram determinados utilizando as seguintes condições: aparato 1 (cesta, 40 mesh); 100 rpm; 900 mL de água destilada mantida a $37 \pm 0,5^{\circ} \mathrm{C}$. Amostras coletadas em: 5, 7, 10, $15,20,30,40,50$ e 60 minutos e a concentração de cefalexina foi determinada por espectrofotometria UV $(262 \mathrm{~nm})$. A partir dos perfis de dissolução determinou-se: modelo matemático de liberação da cefalexina (primeira-ordem); porcentagem de cefalexina dissolvida em 30 minutos (Q30); constante da velocidade de dissolução ( $k$ ); meia-vida de dissolução $\left(t_{50 \%}\right)$; eficiência de dissolução (ED\%). Os valores Q30 obtidos indicaram que os produtos $A 2$ e $B 2$ se apresentaram de acordo com as especificações farmacopéicas. A comparação entre os perfis indicou diferenças estatisticamente significativa entre os produtos $A 2$ e B1 (análise comparativa dos parâmetros cinéticos), A1 e B2 (análise comparativa pelos fatores de diferença - $\mathrm{f}_{1}$ e similaridade - $\mathrm{f}_{2}$ ) e B1 e B2 (análise comparativa dos parâmetros cinéticos e da ED\%).
Unitermos

- Cefalexina

- Cinética de dissolução

- Eficiência de dissolução

\section{INTRODUÇÃO}

A liberação do fármaco de uma forma farmacêutica sólida pode envolver três etapas fundamentais: desintegração, desagregação e, finalmente, dissolução. Esses processos podem ocorrer simultaneamente, com a conseqüente liberação do fármaco da forma farmacêutica. A velocidade pela qual o processo de dissolução ocorre determinará, portanto, a liberação do fármaco e a sua absor- ção. Em função disso os estudos de dissolução in vitro tornaram-se fundamentais para assegurar a qualidade das formulações na forma sólida de uso oral, bem como para permitir a otimização das mesmas quando em desenvolvimento (Adams et al., 2001; Abuzarur-Aloul et al., 1997; Tingstad, 1978).

Existem três categorias de ensaios de dissolução para medicamentos de liberação imediata, os quais permitem avaliar e comparar a cinética e eficiência de dissolu- 
ção de um determinado produto: ensaio de dissolução de um único ponto; ensaio de dissolução de dois pontos e perfis de dissolução. Os perfis de dissolução, os quais são obtidos a partir da porcentagem dissolvida de fármaco em diferentes tempos de amostragem, permitem uma análise mais conclusiva. Entretanto, a grande dificuldade está em relação à forma como estas curvas serão comparadas (Marcolongo, 2003; Brasil, 2002; Storpirtis, Rodrigues, 1998; Serra, 1998; O’Hara, 1998).

Vários métodos têm sido propostos para a avaliação comparativa entre perfis de dissolução. Estes podem ser classificados em: (1) método baseado na análise de variância (ANOVA); (2) método modelo independente; (3) método modelo dependente. Os métodos baseados na análise de variância determinam, através de repetidos ensaios, as fontes de variação (tempo, produto e interação entre tempo versus produto) (Yuksel et al., 2000). Dentre os métodos designados de modelo independente destacam-se os testes combinados como os fatores $f_{l}$ (fator de diferença) e $f_{2}$ (fator de similaridade), que comparam diretamente a diferença entre a porcentagem de fármaco dissolvida em uma unidade de tempo para os produtos teste e referência. Por sua vez, os métodos modelo dependente são baseados em funções matemáticas distintas que, após seleção de uma função adequada, permitem a avaliação dos perfis em relação aos parâmetros derivados dos modelos aplicados. Os exemplos mais comuns encontrados na literatura são: ordem zero, primeira ordem, HixonCrowell, Higushi, quadrático, Weibull, entre outros (Polli, 1996; O’Hara et al., 1998)

Modelos que se fundamentam em estudos da cinética de dissolução possibilitam conclusões a respeito do processo de dissolução de uma determinada formulação, uma vez que permitem conhecer: a velocidade do processo; a quantidade máxima dissolvida e pontos nos quais podem ocorrer mudanças significativas da dissolução (O'Hara et al., 1998; Polli et al., 1996; Moore, Flanner, 1996). Nesse tipo de análise, avalia-se a quantidade de fármaco dissolvida em diferentes intervalos de tempo, obtendo-se um perfil definido por tempo versus porcentagem de fármaco dissolvida. Vários parâmetros cinéticos podem ser descritos a partir desta relação, tais como: constante de velocidade de dissolução (k); meia-vida de dissolução $\left(\mathrm{t}_{50 \%}\right)$; e, quantidade dissolvida em determinado tempo (Q) (O'Hara et al., 1998; Polli et al., 1996; Cid, 1981).

Além dos modelos cinéticos traçados a partir do perfil de dissolução, a eficiência de dissolução (ED\%) sugerida inicialmente por Khan e Rhodes (1975) pode ser incluída como um importante parâmetro de cinética de dissolução. Este termo pode ser definido como a área sob a curva de dissolução em um determinado intervalo de tempo. AED\% permite a comparação mais fidedigna entre dois produtos. Além disso, este parâmetro está relacionado com a quantidade real de fármaco que se encontra dissolvida no meio e, desta forma, pode-se ter um melhor prognóstico dos resultados in vivo (Skoug et al., 1997; Ofoefule et al., 2001).

A cefalexina é uma cefalosporina de primeira geração, pertencente ao grupo dos antibióticos $\beta$-lactâmicos clássicos, que apresentam as mesmas características estruturais das penicilinas. É um antibiótico de amplo espectro, largamente utilizado no Brasil e comercializado na forma de comprimido revestido, por diferentes laboratórios farmacêuticos (DEF, 2004/05). A cefalexina é levemente solúvel na água e possui alta permeabilidade, o que a caracteriza como um antibiótico pertencente à classe 2 da classificação biofarmacêutica (Kasim et al., 2004).

Dentro desse contexto, o presente trabalho teve por objetivo comparar os perfis de dissolução da cefalexina obtidos a partir de produtos distintos, sob a forma de comprimidos revestidos de liberação convencional, utilizando para isto diferentes métodos, tais como: estudos de cinética de dissolução e eficiência de dissolução.

\section{MATERIAL E MÉTODOS}

\section{Perfil de dissolução}

Foram avaliadas duas especialidades farmacêuticas do comércio nacional, sob a forma de comprimidos revestidos, contendo teor declarado de $500 \mathrm{mg}$ de cefalexina base, provenientes de dois laboratórios farmacêuticos distintos. Tais produtos foram designados como A e B, sendo que foram analisados dois lotes distintos ( 1 e 2 ) de cada um deles.

Os perfis de dissolução foram construídos a partir da quantificação da cefalexina dissolvida nas cubas de dissolução, em intervalos de tempo pré-definidos, para os dois lotes dos produtos A e B. As concentrações de cefalexina dissolvida no meio foram obtidas por comparação dos valores de absorvância, determinados por espectrofotometria em comprimento de onda de $262 \mathrm{~nm}$, com a curva de calibração, obtida a partir das soluçõespadrão preparadas com padrão de referência (potência igual a $954,06 \mathrm{mg} / \mathrm{mg}$ ). O equipamento utilizado (Hanson Research Corp., modelo $72 \mathrm{RL}$ ) obedece às especificações da Farmacopéia Brasileira (1988) e da United States Pharmacopeia (2005). As condições utilizadas se encontram descritas no Quadro 1. 
QUADRO 1 - Especificações para a determinação do perfil de dissolução da cefalexina

\begin{tabular}{lc}
\hline Temperatura & $37 \pm 0,5^{\circ} \mathrm{C}$ \\
Sistema de agitação & 1 (cesta -40 mesh) \\
Velocidade & $100 \mathrm{rpm}$ \\
Meio de dissolução & água degaseificada \\
Volume do meio & $900 \mathrm{~mL}$ \\
Alíquota amostrada & $10 \mathrm{~mL}$ \\
Tempo de amostragem & $5,7,10,15,20,30$, \\
& 40,50 e 60 minutos \\
Detecção & Espectrofotometria \\
& $\mathrm{UV}(262 \mathrm{~nm})$ \\
\hline
\end{tabular}

Para a realização do perfil de dissolução dos produtos em questão, para cada produto, foram utilizados 6 comprimidos de cada lote. Padronizou-se a coleta de alíquotas $(10,0 \mathrm{~mL})$ do meio nos tempos: 5, 7, 10, 15, 20, 30, 40, 50 e 60 minutos, e, a cada tomada de ensaio, o meio de dissolução (água a $37^{\circ} \mathrm{C}$ ) foi reposto. As alíquotas amostradas foram centrifugadas e fração do sobrenadante foi diluída adequadamente com água destilada para a análise espectrofotométrica, que foi realizada em comprimento de onda de $262 \mathrm{~nm}$ (UV), segundo metodologia preconizada pela United States Pharmacopeia (2005) e validada. Os valores obtidos para cada intervalo de coleta foram corrigidos considerando-se as alíquotas retiradas e expressos através de porcentagem em função do tempo (Aronson, 1993).

\section{Cinética de dissolução}

Os resultados obtidos a partir do perfil de dissolução foram submetidos a cálculos matemáticos e estatísticos com o objetivo de determinar a cinética de dissolução da cefalexina para os produtos A e B. A equação de regressão da reta foi determinada através das porcentagens dissolvidas em função do tempo, utilizando-se dos modelos cinéticos de ordem zero e de primeira-ordem (Nikolic et al., 1992; Shin, Cho, 1996; Cid, 1981). Para avaliar os perfis de dissolução através dos modelos propostos, estabeleceram-se as seguintes relações:

Modelo de ordem zero - para cada produto construiu-se um gráfico que relacionou tempo (minutos) versus quantidade da cefalexina não dissolvida (quantidade total - $Q ¥$ subtraída da quantidade dissolvida de cefalexina - Q).

Modelo de primeira-ordem - para cada produto construiu-se um gráfico que relacionou tempo (minutos) versus logaritmo neperiano da porcentagem de cefalexina não dissolvida $(\ln \% \mathrm{ND})$ em função do tempo (minutos).
O modelo matemático que melhor expressou o perfil de dissolução da cefalexina dos produtos foi selecionado após a análise de regressão linear através da avaliação estatística por análise de variância One-Way (ANOVA), estabelecendo-se o índice de correlação (r) e o nível de significância da correlação através dos valores de $p$ e de $F$.

As constantes cinéticas da dissolução foram determinadas a partir das equações definidas pelo modelo matemático que apresentou índice de correlação maior e mais significativo. Dentre os parâmetros cinéticos foram calculados:

- Meia-vida de dissolução $\left(t_{50 \%}\right)$ : tempo no qual se dissolvem $50 \%$ da cefalexina. Este parâmetro foi calculado após a obtenção dos valores de $\mathrm{k}$, em função do modelo cinético do processo e a partir da seguinte equação:

$$
\mathrm{t}_{50 \%}=\frac{0,693}{\mathrm{k}}
$$

- Quantidade dissolvida de cefalexina após 30 minutos do início do ensaio de dissolução (Q30): este valor foi definido matematicamente conforme a equação da reta de regressão que melhor definiu o perfil de dissolução. De acordo com a United States Pharmacopeia (2005) não deve ser inferior a $80 \%$ da quantidade total declarada no rótulo;

- Constante de velocidade de dissolução (k): foi calculada após a seleção do modelo cinético mais adequado para cada produto. Corresponde ao coeficiente angular da inclinação da reta de regressão.

Os resultados referentes a: $\mathrm{t}_{50 \%}, \mathrm{Q} 30 \mathrm{e} \mathrm{k}$, foram submetidos à análise de variância One-Way (ANOVA) e, com a finalidade de determinar diferenças entre as médias obtidas a partir dos produtos A e B, lotes 1 e 2, foi aplicado o teste-t de Dunnett. Valores de $\mathrm{p}<0,05$ foram considerados estatisticamente significativos (Bolton, 1997).

\section{Eficiência de dissolução (ED\%)}

Foi calculada a partir dos valores obtidos de área sob a curva (ASC) do perfil de dissolução da cefalexina no intervalo de tempo $(\mathrm{t})$, através do método dos trapezóides (Khan, Rhodes, 1975). Determinou-se a ED através da razão entre a área sob a curva de dissolução da cefalexina no intervalo de tempo compreendido entre zero e 60 minutos $\left(\mathrm{ASC}_{0-60 \text { minutos }}\right)$ e a área total do retângulo $\left(\mathrm{ASC}_{\mathrm{TR}}\right)$ definido pela ordenada ( $100 \%$ de dissolução) e pela abcissa (tempo igual a 60 minutos). A ED foi expressa em porcen- 
tagem e pode ser definida pela seguinte equação:

$$
\mathrm{ED}=\frac{\mathrm{ASC}_{(0-60)}}{\mathrm{ASC}_{\mathrm{TR}}} \times 100 \%
$$

Os resultados referentes a $\mathrm{ED} \%$ para os produtos $\mathrm{A}$ e $\mathrm{B}$, lotes 1 e 2, foram submetidos à análise de variância One-Way (ANOVA) e, com a finalidade de determinar diferenças entre as médias obtidas, foi aplicado o teste- $\mathrm{t}$ de Dunnett. Valores de $\mathrm{p}<0,05$ foram cosiderados estatisticamente significativos (Bolton, 1997).

\section{Fatores de diferença $\left(f_{1}\right)$ e similaridade $\left(f_{2}\right)$}

A comparação dos perfis obtidos no presente estudo foi também realizada através da determinação dos fatores $f_{I}$ e $f_{2}$ (Moore, Flanner, 1996; FDA Guidance, 1997). O fator de diferença $\left(f_{l}\right)$ indica a porcentagem de diferença entre os dois perfis de dissolução e pode ser calculado através da seguinte fórmula:

$$
\left.f_{1}=\left\{\left[\sum \mathrm{t}=1^{\mathrm{n}}|\mathrm{Rt}-\mathrm{Tt}|\right]\right\} /\left[\sum \mathrm{t}=1^{\mathrm{n}} \mathrm{Rt}\right]\right\} * 100
$$

Onde: $\mathbf{n}=$ número do tempo; $\mathbf{R t}=$ valor de referência da dissolução do grupo de tempo t; $\mathbf{T t}=$ valor do teste de dissolução do grupo de tempo $t$

O fator de similaridade $\left(f_{2}\right)$ é um logarítmo recíproco da transformação da raiz quadrada da soma do erro quadrado e indica a média da similaridade da porcentagem de dissolução entre os dois perfis. É calculado de acordo com a fórmula abaixo:

$$
f_{2}=50 * \log \left\{\left[(1+1 / \mathrm{n}) \sum \mathrm{t}=1^{\mathrm{n}}(\mathrm{Rt}-\mathrm{Tt})^{2}\right]^{-0,5 * 100}\right\}
$$

Onde: $\mathbf{n}=$ número do tempo; $\mathbf{R t}=$ valor de referência da dissolução do grupo de tempo t; $\mathbf{T t}=$ valor do teste de dissolução do grupo de tempo t.

De acordo com os dados da literatura a semelhança ou equivalência de dois perfis é observada quando os valores de $f_{1}$ se apresentarem entre 0 e 15 e os de $f_{2}$, entre 50 e 100 (Moore, Flanner, 1996; FDA Guidance, 1997).

\section{RESULTADOS E DISCUSSÃO}

As características biofarmacotécnicas de formas farmacêuticas sólidas são principalmente delineadas pelo ensaio de dissolução in vitro, uma vez que este processo é, geralmente, etapa limitante para a absorção de fármacos. Assim, uma abordagem precisa em relação ao perfil e cinética do processo de dissolução permite a obtenção de dados mais conclusivos em relação às formulações em estudo (Dressman et al., 1998; Abdou, 1995; Tingstad, 1978).

Os perfis de dissolução dos produtos A e B, lotes 1 e 2 , foram obtidos através de coletas seriadas em intervalos de tempo pré-definidos de acordo com Quadro 1 e estão ilustrados na Figura 1. Os valores médios com os respectivos desvios-padrão para cada um dos produtos avaliados encontram-se na Tabela I. A comparação dos perfis de dissolução obtidos a partir dos produtos avaliados no pre-

\begin{tabular}{|c|c|c|c|c|}
\hline $\begin{array}{l}\text { Tempo } \\
\text { (minutos) }\end{array}$ & A $1(\%)$ & A $2(\%)$ & B $1(\%)$ & B $2(\%)$ \\
\hline 5 & $4,74 \pm 0,82$ & $2,97 \pm 0,45$ & $5,65 \pm 2,18$ & $5,91 \pm 3,68$ \\
\hline 7 & $17,19 \pm 3,16$ & $9,10 \pm 3,96$ & $19,33 \pm 6,76$ & $15,71 \pm 8,76$ \\
\hline 10 & $26,06 \pm 5,24$ & $27,45 \pm 4,07$ & $33,23 \pm 6,75$ & $34,64 \pm 12,17$ \\
\hline 15 & $39,28 \pm 7,37$ & $47,72 \pm 5,26$ & $53,40 \pm 7,88$ & $59,54 \pm 9,70$ \\
\hline 20 & $49,59 \pm 8,24$ & $65,14 \pm 6,33$ & $63,46 \pm 7,13$ & $72,61 \pm 9,62$ \\
\hline 30 & $72,84 \pm 10,13$ & $86,68 \pm 7,63$ & $77,04 \pm 4,62$ & $86,16 \pm 8,49$ \\
\hline 40 & $85,28 \pm 10,45$ & $91,18 \pm 4,41$ & $84,69 \pm 3,77$ & $91,35 \pm 5,78$ \\
\hline 45 & $90,37 \pm 9,26$ & $94,17 \pm 4,34$ & $86,48 \pm 3,29$ & $93,53 \pm 5,36$ \\
\hline 50 & $95,37 \pm 10,11$ & $97,34 \pm 5,37$ & $87,75 \pm 2,99$ & $95,48 \pm 5,78$ \\
\hline 60 & $99,61 \pm 8,30$ & $99,60 \pm 3,95$ & $88,86 \pm 3,08$ & $98,09 \pm 3,54$ \\
\hline
\end{tabular}

TABELA I - Valores das médias das porcentagens de cefalexina dissolvida ( \pm desvios-padrão) em função do tempo, a partir dos produtos A e B (lotes 1 e 2)

Os valores correspondem à média de seis determinações \pm desvio-padrão. No ensaio do perfil de dissolução empregouse: meio de dissolução = água destilada e desgaseificada; aparato = 1 (cesta -40 mesh); volume do meio $=900 \mathrm{~mL}$; velocidade de agitação $=100 \mathrm{rpm}$; leitura $=262 \mathrm{~nm}$. 


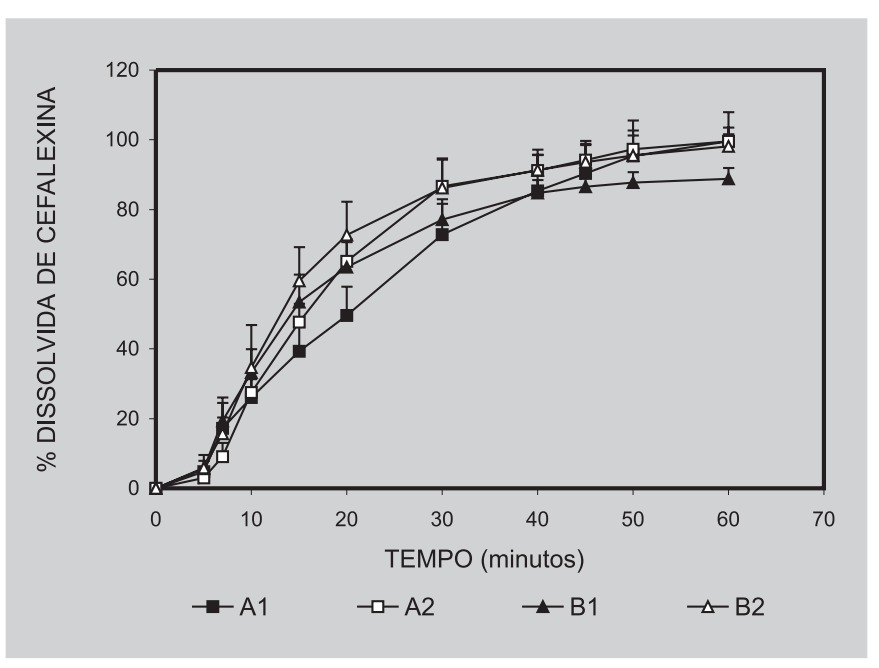

FIGURA 1 - Perfis de dissolução da cefalexina a partir dos comprimidos (500 mg), produtos A e B (lotes 1 e 2), em água a $37^{\circ} \mathrm{C}$, velocidade de agitação a $100 \mathrm{rpm}$. Os valores correspondem à média de seis determinações. As barras representam os desvios-padrão.

sente estudo foi estabelecida através de: estudo de cinética de dissolução, eficiência de dissolução (ED\%) e fatores $f_{l}$ e $f_{2}$.

A partir dos perfis de dissolução obtidos, que relacionam porcentagem dissolvida versus tempo, determinaram-se parâmetros cinéticos, os quais possibilitaram análise comparativa com relação ao comportamento in vitro desses produtos. A avaliação da cinética permite conclusões a respeito do processo de dissolução de determinada formulação, uma vez que possibilita conhecer: (i) a velocidade do processo; (ii) a quantidade máxima dissolvida e (iii) os pontos nos quais podem ocorrer mudanças significativas da dissolução (Marcolongo, 2003; Abdou, 1995; Dokoumetzidis et al., 2006).

Alguns autores têm utilizado modelo de primeira ordem, entre outros, como método de comparação modelo dependente de perfis de dissolução de comprimidos de liberação imediata. Para aplicar um método de comparação modelo dependente, a primeira etapa é a determinação do modelo cinético que melhor representa o processo de dissolução. Esta definição é obtida através da análise da regressão linear dos resultados obtidos a partir do perfil de dissolução, empregando-se modelos matemáticos, tais como: ordem zero, primeira ordem, entre outros. Os valores de coeficiente de correlação $(r)$ e os índices de significância $(F$ e $p)$, obtidos na análise da regressão linear, indicarão o modelo cinético que melhor se ajusta ao perfil de dissolução dos produtos avaliados (Yuksel et al, 2000; Bravo et al., 2004; Dokoumetzidis et al., 2006).

No presente estudo, os coeficientes de correlação $(r)$ para os produtos A e B (lotes 1 e 2) ficaram entre 0,9971 e 0,9989 e os valores de $F$ e $p$ indicaram um alto grau de significância para o modelo de primeira ordem (Tabela II). Estes resultados estão de acordo com aqueles descritos na literatura, uma vez que este modelo está principalmente relacionado às formulações de liberação convencional (Ishii et al., 1996; Cid, 1981). As equações obtidas para os produtos analisados estão descritas na Tabela III e as Figuras 2 e 3 apresentam os perfis resultantes da aplicação de ambos os modelos matemáticos (ordem zero e primeira ordem, respectivamente) para os valores médios de dissolução dos produtos A e B (lotes 1 e 2).

TABELA II - Parâmetros estatísticos dos estudos de regressão, aplicando modelo de zero ordem e primeira ordem, derivados dos perfis de dissolução dos produtos $\mathrm{A}$ e B (lotes 1 e 2)

\begin{tabular}{lcccc}
\hline Modelo & \multicolumn{2}{c}{ Ordem Zero } & \multicolumn{2}{c}{ Primeira } \\
\hline & $\mathrm{r}$ & $\mathrm{F}$ & $\mathrm{r}$ & $\mathrm{F}$ \\
$\mathrm{A} 1$ & $0,9825 *$ & 269,78 & $0,9986 *$ & 568,60 \\
$\mathrm{~A} 2$ & $0,9614 *$ & 83,77 & $0,9989 *$ & 342,18 \\
$\mathrm{~B} 1$ & $0,9345 *$ & 48,76 & $0,9986 *$ & 518,91 \\
$\mathrm{~B} 2$ & $0,9532 *$ & 60,05 & $0,9971 *$ & 543,97 \\
\hline
\end{tabular}

$\mathrm{r}=$ índice de correlação; $\mathrm{F}=$ relação de variância maior $\mathrm{e}$ menor; ${ }^{*} p<0,0001$

A partir do modelo de primeira-ordem foram determinados os seguintes parâmetros cinéticos: constante de velocidade de dissolução (k); meia-vida de dissolução $\left(\mathrm{t}_{50 \%}\right)$; quantidade dissolvida em 30 minutos do ensaio de

TABELA III - Equações obtidas a partir dos perfis de dissolução dos produtos A e B (lotes 1 e 2) segundo cinética de primeira ordem

\begin{tabular}{lll}
\hline Lotes & A & B \\
\hline 1 & $\ln \% \mathrm{ND}=4,82( \pm 0,18)-0,05( \pm 0,01) \mathrm{t}$ & $\ln \% \mathrm{ND}=4,65( \pm 0,15)-0,04( \pm 0,00) \mathrm{t}$ \\
2 & $\ln \% \mathrm{ND}=4,98( \pm 0,18)-0,07( \pm 0,01) \mathrm{t}$ & $\ln \% \mathrm{ND}=4,89( \pm 0,16)-0,07( \pm 0,02) \mathrm{t}$ \\
\hline
\end{tabular}

$\ln \% \mathrm{ND}=$ logaritmo neperiano da porcentagem de fármaco não-dissolvida; $\mathrm{t}=$ tempo (minutos). Entre parênteses estão os respectivos desvios-padrão 


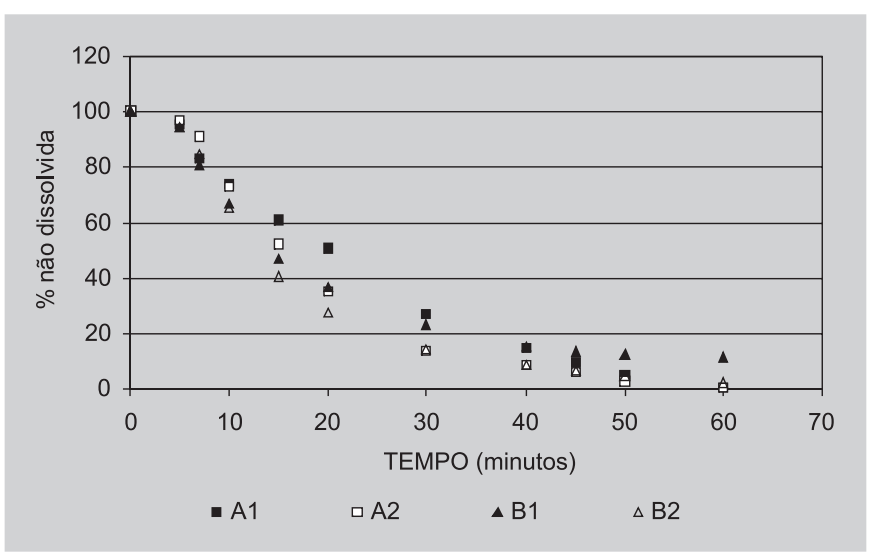

FIGURA 2 - Porcentagem de cefalexina não dissolvida a partir dos comprimidos (500 mg), produtos A e B (lotes 1 e 2), em água a $37^{\circ} \mathrm{C}$, velocidade de agitação a $100 \mathrm{rpm}$. Os valores correspondem à média de seis determinações. Aplicação do modelo de ordem zero.

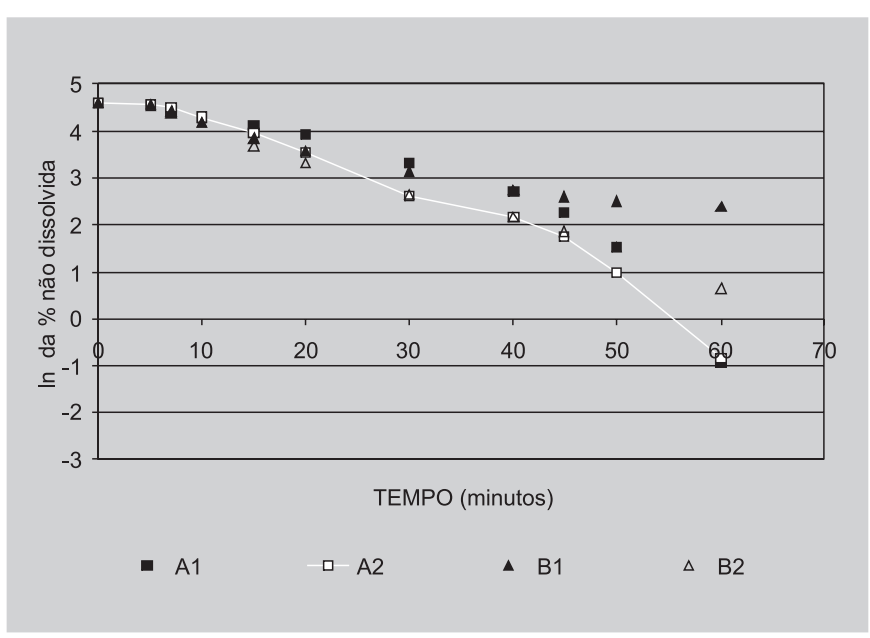

FIGURA 3 - Transformação logarítmica da porcentagem de cefalexina não dissolvida a partir dos comprimidos (500 mg), produtos A e B (lotes 1 e 2), em água a $37^{\circ} \mathrm{C}$, velocidade de agitação a $100 \mathrm{rpm}$. Os valores correspondem à média de seis determinações. Aplicação do modelo de primeira ordem. dissolução (Q 30). Os resultados indicaram que os perfis de dissolução dos produtos A e B foram diferenciados, principalmente, nos 30 minutos iniciais do ensaio de dissolução. Todavia, os valores de $t_{50 \%}, \mathrm{Q} 30$ e $k$ apontaram para melhor desempenho dos produtos A2 e B2 (Tabela IV).

Os valores de Q30 indicaram maior porcentagem de dissolução para A2 $(83,21 \pm 5,76 \%)$ e B2 $(84,33 \pm 8,96 \%)$, ambos cumpriram com as especificações exigidas pela United States Pharmacopeia (2005), a qual especifica que, após o intervalo de 30 minutos, os produtos devem liberar, no mínimo, $80 \%$ do valor declarado no rótulo. O mesmo não foi observado para os produtos A1 $(72,62 \pm 9,77 \%)$. e B1 $(73,32 \pm 3,20 \%)$. A comparação dos parâmetros $t_{50 \%}$ e Q30, apontou para diferenças estatisticamente significativas entre A2 e B1 $(\mathrm{p}<0,05)$ e B1 e B2 $(\mathrm{p}<0,05)($ Tabela VI).

Outro método de comparação de perfis de dissolução baseia-se no cálculo de eficiência de dissolução (ED\%), estabelecida por Khan e Rhodes (1975). A ED\% pode ser definida como área sob a curva de dissolução em um tempo específico e é expressa como percentual da área do retângulo descrito por $100 \%$ de dissolução nesse tempo. Esse parâmetro está relacionado com a quantidade real de fármaco dissolvida no meio e, desta forma, pode-se ter um melhor prognóstico dos resultados in vivo.

Alguns pesquisadores têm defendido a utilização deste recurso matemático para comparar formulações farmacêuticas, uma vez que a biodisponibilidade é estimada por integração da área sob a curva de concentrações plasmáticas e os resultados de dissolução in vitro são expressos através da área sob a curva de concentrações dissolvidas do fármaco (Ofoefule et al., 2001; Khan, Rhodes, 1975).

Os resultados do percentual de eficiência de dissolução estão apresentados na Tabela V. A comparação estatística entre as médias de ED\% realizada através do teste-t de Dunnett indicou diferenças significativas apenas entre B1 e B2 $(\mathrm{p}<0,05)$. Não foram observadas diferenças estatisticamente significativas com relação a ED\% dos

TABELA IV - Valores médios dos parâmetros cinéticos (desvios-padrão) a partir do modelo de cinética de primeira ordem, derivados dos perfis de dissolução dos produtos A e B (lotes 1 e 2)

\begin{tabular}{lccc}
\hline Produtos & $\mathrm{t}_{50 \%}(\mathrm{~min})$ & $\mathrm{Q} \mathrm{30}(\%)$ & $\mathrm{k}\left(\mathrm{min}^{\mathrm{c}}\right)$ \\
\hline A1 & $14,22( \pm 4,40)$ & $72,62( \pm 9,77)$ & $0,05( \pm 0,01)$ \\
$\mathrm{A} 2$ & $10,18( \pm 2,05)$ & $83,21( \pm 5,76)$ & $0,07( \pm 0,01)$ \\
$\mathrm{B} 1$ & $15,85( \pm 1,01)$ & $73,32( \pm 3,20)$ & $0,04( \pm 0,00)$ \\
B2 & $10,00( \pm 3,17)$ & $84,33( \pm 8,96)$ & $0,07( \pm 0,02)$ \\
\hline
\end{tabular}

Os valores representam os parâmetros cinéticos calculados, em que: $\mathrm{k}=$ constante de velocidade de dissolução $\left(\mathrm{min}^{-1}\right) ; \mathrm{t} 50 \%=$ tempo no qual se dissolvem $50 \%$ da cefalexina $(\mathrm{min}) ; \mathrm{Q} 30=$ quantidade de cefalexina dissolvida aos 30 minutos $(\%)$ 
TABELA V - Eficiência de dissolução (ED\%) dos produtos A e B (lotes 1 e 2)

\begin{tabular}{lcc}
\hline Lotes & A (\%) & B (\%) \\
\hline $\mathbf{1}$ & $62,80 \pm 8,45$ & $64,74 \pm 4,57$ \\
$\mathbf{2}$ & $68,69 \pm 4,18$ & $71,03 \pm 6,63$ \\
\hline
\end{tabular}

Os valores representam a média \pm desvio-padrão

produtos $\mathrm{A} 2$ e $\mathrm{B} 1$, fato observado pela comparação dos parâmetros cinéticos ( $\mathrm{t}_{50 \%}$ e Q30).

Os fatores $f_{1}$ e $f_{2}$ foram calculados segundo os critérios estabelecidos pela literatura, entretanto, o mesmo foi conduzido avaliando-se seis amostras de cada produto (A e B), lotes (1 e 2). A semelhança ou equivalência de dois perfis é observada quando os valores de $f_{1}$ se apresentarem entre 0 e 15 e os de $f_{2}$ entre 50 e 100 (Moore, Flanner, 1996; Shah et al., 1998; FDA Guidance, 1997). Os resultados obtidos indicaram diferenças entre os produtos A1 e $\mathrm{B} 2\left(f_{1}-25,05 ; f_{2}-47,30\right)$. Em relação ao produto A, lotes $1 \mathrm{e} 2$, o valor de $f_{l}$ ficou acima do especificado $(17,62)$.

Os resultados obtidos pelas comparações dos parâmetros cinéticos $\left(\mathrm{k}\right.$; $\mathrm{t}_{50 \%}$ e Q30) e dos valores da eficiência de dissolução (ED\%) mostraram concordância apenas com relação ao produto B (lotes 1 e 2), pois ambos indicaram diferenças significativas entre os dois lotes do mesmo. Por outro lado, esta diferença não foi detectada pela aplicação do modelo independente $f_{1}$ e $f_{2}$.

Como a cefalexina pertence à classe 2 da classificação biofarmacêutica (Kasim et al., 2004), cabe ressaltar que a dissolução in vivo é a etapa limitante da absorção e esta pode ser variável devido aos fatores relacionados às formulações. Assim, os estudos de dissolução in vitro são fundamentais para assegurar a qualidade das formulações na forma sólida de uso oral, bem como para permitir a otimização das mesmas quando em desenvolvimento. Portanto, a definição do método utilizado para a comparação dos perfis obtidos deve ser cuidadosa.

Os resultados obtidos no presente estudo revelam que distintos métodos de comparação podem originar diferentes resultados. Segundo Yuksel et al. (2000), os métodos modelo independente $\left(f_{1}\right.$ e $\left.f_{2}\right)$ são menos discriminatórios que os métodos modelo dependente e do que aqueles baseados na análise de variância (ANOVA). Portanto, a aplicação de diferentes métodos na comparação dos perfis de dissolução pode fornecer informaçoes mais precisas sobre o comportamento de dissolução dos produtos em análise e facilitar o desenvolvimento ou otimização de formulações.

\section{CONCLUSÕES}

1. De acordo com os dados estatísticos obtidos, os perfis de dissolução de ambos os produtos (A e B) seguiram

TABELA VI - Índices de significância (p) resultantes das comparações entre os produtos A e B (lotes 1 e 2) para os seguintes parâmetros: $\mathrm{t}_{50 \%}(\mathrm{~min}) ; \mathrm{Q} 30(\%) ; \mathrm{k}\left(\min ^{\prime-1}\right)$ e ED\%

\begin{tabular}{|c|c|c|c|c|c|c|c|c|c|c|c|c|}
\hline & \multicolumn{4}{|c|}{$\mathrm{A} 2$} & \multicolumn{4}{|c|}{ B1 } & \multicolumn{4}{|c|}{ B2 } \\
\hline & $\begin{array}{c}\mathrm{t}_{50 \%} \\
(\mathrm{~min})\end{array}$ & $\begin{array}{l}\text { Q 30 } \\
(\%)\end{array}$ & $\begin{array}{c}\mathrm{k} \\
\left(\mathrm{min}^{-1}\right)\end{array}$ & $\mathrm{ED} \%$ & $\begin{array}{c}\mathrm{t}_{50 \%} \\
(\mathrm{~min})\end{array}$ & $\begin{array}{l}\text { Q 30 } \\
(\%)\end{array}$ & $\begin{array}{c}\mathrm{k} \\
\left(\mathrm{min}^{-1}\right)\end{array}$ & ED\% & $\begin{array}{c}\mathrm{t}_{50 \%} \\
(\mathrm{~min})\end{array}$ & $\begin{array}{l}\text { Q 30 } \\
(\%)\end{array}$ & $\begin{array}{c}\mathrm{k} \\
\left(\mathrm{min}^{-1}\right)\end{array}$ & $\mathrm{ED} \%$ \\
\hline A1 & 0,073 & 0,072 & 0,011 & 0,205 & 0,395 & 0,877 & 0,130 & 0,795 & 0,121 & 0,086 & 0,051 & 0,083 \\
\hline A2 & & & & & 0,000 & 0,005 & 0,000 & 0,119 & 0,885 & 0,819 & 0,909 & 0,230 \\
\hline B 1 & 0,000 & 0,005 & 0,000 & 0,119 & & & & & 0,002 & 0,020 & 0,003 & 0,041 \\
\hline B 2 & 0,885 & 0,819 & 0,909 & 0,230 & 0,002 & 0,020 & 0,003 & 0,041 & & & & \\
\hline
\end{tabular}

Valores de $\mathrm{p}<0,050$ foram considerados estatisticamente significativos

TABELA VII - Valores de $f_{l}$ e $f_{2}$ resultantes das comparações entre os produtos A e B (lotes 1 e 2 )

\begin{tabular}{lcccccc}
\hline & \multicolumn{2}{c}{ A2 } & \multicolumn{2}{c}{ B1 } & \multicolumn{2}{c}{ B2 } \\
\cline { 2 - 7 } & $f_{1}$ & $f_{2}$ & $f_{1}$ & $f_{2}$ & $f_{2}$ \\
\hline A1 & 17,62 & 52,76 & 12,17 & 55,32 & 25,05 & 47,30 \\
A2 & & & 11,97 & 65,87 & 11,82 & 58,74 \\
B 1 & 11,97 & 65,87 & & & 10,14 & 58,47 \\
B2 & 11,82 & 58,74 & 10,14 & 58,47 & & \\
\hline
\end{tabular}


cinética de dissolução de primeira ordem;

2. Os valores de $\mathrm{Q} 30$ indicaram maior porcentagem de dissolução para A2 $(83,21 \pm 5,76 \%)$ e para B2 $(84,33$ $\pm 8,96 \%$ );

3. O percentual total de cefalexina dissolvida, para os produtos $\mathrm{A} 2$ e B2, aos 30 minutos, cumpriu com as especificações farmacopéicas, o que não foi observado com A1 e B1;

4. A comparação dos parâmetros $\mathrm{t}_{50 \%}$ e Q30 e obtidos a partir dos produtos A e B, lotes 1 e 2, apontou para diferenças estatisticamente significativas entre A2 e B1 $(\mathrm{p}<0,05)$ e B1 e B2 $(\mathrm{p}<0,05)$;

5. A comparação estatística entre as médias de $\mathrm{ED} \%$ indicou diferenças significativas entre B1 e B2 $(\mathrm{p}<0,05)$;

6. Os resultados de $f_{1}$ e $f_{2}$ indicaram diferenças entre os produtos $\mathrm{A} 1$ e $\mathrm{B} 2\left(f_{1}-25,05 ; f_{2}-47,30\right)$.

7. Os resultados obtidos pelas comparações através de modelo dependente e da eficiência de dissolução (ED\%) mostraram concordância apenas com relação ao produto $\mathrm{B}$ (lotes 1 e 2), pois ambos indicaram diferenças significativas entre os dois lotes do mesmo.

8. Esta diferença não foi detectada pela aplicação do modelo independente $f_{1}$ e $f_{2}$

\section{ABSTRACT}

\section{Dissolution profile comparison of cephalexin by dissolution kinetic studies and dissolution efficiency (ED\%)}

The present study reports the comparative results of the cephalexin dissolution kinetic evaluation and dissolution efficiency (ED\%) considering two brands (1 and 2) of different products (A and B), available within the Brazilian market in the tablet conventional release dosage form. The dissolution profiles have been determined adopting the following conditions: apparatus 1 (basket, 40 mesh); $100 \mathrm{rpm} ; 900 \mathrm{~mL}$ of distilled water kept $37 \pm 0,5{ }^{\circ} \mathrm{C}$. Samples have been collected in: 5, 7, 10, 15, 20, 30, 40, 50 and 60 minutes and the cephalexin concentration was determined at UV spectrofotometry $(262 \mathrm{~nm})$. Through the dissolution profiles it has been determined: (i) the mathematical model of cephalexin released (firstorder); (ii) the percentage of cephalexin dissolved at 30 minutes (Q30); (iii) the constant of the dissolution rate ( $k$ ); (iv) half-life of dissolution (t50\%); and, (v) the efficiency of dissolution (ED\%). The percentage of cephalexin dissolved at 30 minutes (Q30) has indicated that the products $A 2$ e $B 2$ were in accordance with the presented pharmacopoeia specifications. Dissolution profile comparison has indicated significant statistic difference between: $A 2$ and $B 1$ (comparative analysis of the kinetic parameters), $A 1$ and $B 2$ (comparative analysis by difference factor $-f_{1}$ and similarity factor $-f_{2}$ ), B1 and B2 (comparative analysis of the kinetic parameters and $E D \%)$.

UNITERMS: Cephalexin. Dissolution kinetics. Dissolution effectiveness.

\section{REFERÊNCIAS BIBLIOGRÁFICAS}

ABDOU, H. M. Dissolution. In: REMINGTON'S pharmaceutical sciences. 19. ed. Easton: Mack, 1995. p. 593-604.

ABUZARUR-ALOUL, R.; GJELLAN, K.; SJOLUND, M.; LOFQVIST, M.; GRAFFNER, C. Critical dissolution tests of oral systems based on statistically designed experiments. Screning of critical fluids and in vitro/in vivo modelling of extended release coated spheres. Drug Dev. Ind. Pharm., New York, v. 23, n. 8, p. 749-760, 1997.

ADAMS, E., COOMANS, D., SMEYERS-VERBEKE, J., MASSART, D. L. Application of linear mixed effects models to the evaluation of dissolution profiles. Int. J. Pharm., Amsterdam, v.226, p.107-125, 2001.

AOKI, H., OKUHARA, M. Natural b-lactam antibiotics. Annu. Rev. Microbiol., Palo Alto, v. 34, p. 159-181, 1980.

ARONSON, H. Correction factor for dissolution profile calculations. J. Pharm. Sci., Washington, v. 82, n. 11, p. $1190,1993$.

AULTON, M. E. Pharmaceutics: the science of dosage form design. New York: Churchi Livingstone, 1996. 734 p.

BOLTON, S. Pharmaceutical statistics, practical and clinical applications. New York: Marcel Dekker, 1997. p. 148-156.

BRASIL. Resolução RE n.484, de 19 de março de 2002. A Agência Nacional de Vigilância Sanitária aprova guia para ensaios de dissolução para formas farmacêuticas sólidas de liberação imediata (FFSLI) Diário Oficial da União, Brasília, 20 mar. 2002. Seção 1, p.116-118. 
BRAVO, S.A; LAMAS, M.C.; SALOMON, C.L. Swellable matrices for the contolled-release of diclofenac sodium: formulation and in vitro studies. Pharm. Dev. Technol., v. 9, n. 1, p. 75-83, 2004.

CID, E.C. Cinética de dissolución de medicamentos. Washington: Organización de los Estados Americanos, 1981. $102 \mathrm{p}$.

DICIONÁRIO de Especialidades Farmacêuticas. 29 ed. Rio de Janeiro: Publicações Científicas, 2004. 898 p.

DOKOUMETZIDIS, A; PAPADOPOULOU, V.; MACHERAS, P. Analysis of dissolution data using modified versions of Noyes-Whitney equation and the Weibull function. Pharm. Res., v. 23, n. 2, p. 256-261, 2006.

DRESSMAN, J. B.; AMIDON, G. L.; REPPAS, C., SHAH, V.P. Dissolution testing as a prognostic tool for oral drug absorption: immediate release dosage forms. Pharm. Res., New York, v. 15, n. 1, p. 11-22, 1998.

FARMACOPÉIA Brasileira. 4 ed. São Paulo: Atheneu, 1988. $600 \mathrm{p}$.

FDA Guidance for industry: dissolution testing of immediate release solid oral dosage forms. Rockville: FDA, 1997. p. 1-11.

KAISIM, A.N.; WHITEHOUSE, M.; RAMACHANDRAN, C,; BERMEJO, M.; LENNERNÄS, H.; HUSSAIN, A.S.; JUNGINGER, H.E.; STAVCHANSKY, S.A.; MIDHA, K.K.; SHAH, V.P.; AMIDON, G.L. Molecular properties of WHO essential drugs and provisional biopharmaceutical classification. Mol. Pharm., v.1, n.1, p.85-96, 2004.

KHAN, K. A; RHODES, C. T. The concept of dissolution efficiency. J. Pharm. Pharmacol., London, v.27, p. 4849, 1975.

LIPKA, E., AMIDON, G. L. Setting bioequivalence requiremens for drug development based on preclinical data: optimizing oral drug delivery systems. J. Controlled Release, Shannon, v. 62, p. 41-49, 1999.

MARCOLONGO, R. Dissolução de medicamentos: fundamentos, aplicações, aspectos regulatórios e perspectivas na área farmacêutica. São Paulo, 2003, 114 p. [Dissertação de Mestrado, Faculdade de Ciências Farmacêuticas, Universidade de São Paulo].
MOORE, J.W.; FLANNER, H.H. Mathematical comparison of dissolution profiles. Pharm. Technol., v.20, n.6, p. 6474, 1996.

NIKOLIC, L., DJURIC, Z., JOVANOVIC, M. Influence of in vitro test conditions on release of aspirin from commercial tablets. J. Pharm. Sci., Washington, v. 81, n. 4,p. 386-391, 1992.

OFOEFULE, S.I.; UDEOGARANYA, P.O; OKONTA, J.M. Prediction of in vivo bioavailability of six brands of ciprofloxacin film coated tablets using the concept dissolution efficiency (DE). Boll. Chim. Farm., Milano, v. 140, n.3, p. 187-191, 2001.

O'HARA, T.; DUNNE, A; BUTLER, J.; DENAVE, J. A review of methods used to compare dissolution profile data. Pharm. Sci. Technol. Today, Cambridge, v.1, n. 5, 214-223, 1998.

POLLI, J.E.; REKHI, G.S.; SHAH, V.P. Methods to compare dissolution profiles. Drug Inf. J., v.30, p. 1113 20, 1996

SERRA, C. H. R. Avaliação biofarmacotécnica de comprimidos contendo cefalexina: cinética de dissolução e bioequivalência. São Paulo, 1998. 206 p. [Tese de Doutorado, Faculdade de Ciências Farmacêuticas, Universidade de São Paulo].

SHAH, V.P.; TSONG, Y.; SATHE, P.; LIU, J-P. In vitro dissolution profile comparison - statistics and analysis of the similarity factor f2. Pharm. Res., v. 15, n. 6, p. 889-896, 1998.

SHARGEL, L., YU, A. B. C. Applied biopharmaceutics and pharmacokinetics. 3 ed. Connecticut : Prentice Hall, 1993.625p.

SHIN, S.M., CHO, S-J. Kinetics of cephalexin release from eudragit-hidroxypropylcellulose membranes. Drug Dev. Ind. Pharm., New York, v. 22, n. 4, p. 299-305, 1996.

SKOUG, J. W.; HALSTEAD, G. W.; THIES, D. L.; FREEMAN, J. E.; FAGAM, D. T.; ROHRS, B. R. Roteiro para o desenvolvimento e validação do teste de dissolução em formas farmacêuticas sólidas para uso oral. Pharm. Technol., São Paulo, n. 1, p. 34-43, 1997. 
STORPIRTIS , S.; RODRIGUES , D. - In vitro evaluation of dissolution properties and degradation products of omeprazole in enteric-coated pellests . Drug Dev. Ind. Pharm., Boston, v. 24, n. 11, p. 123-129, 1998.

TINGSTAD, J. E. Dissolution rate testing for the optimization of formulations. Pharm. Ind., Aulendorf, v. 40, n.7, p. 751757,1978 .
UNITED States Pharmacopeia. 28 ed. rev. Rockville: United States Pharmacopeial Convention, 2005. 3000 p.

YUKSEL, N.; KANIK, A E.; BAYKARA, T. Comparison of in vitro dissolution profiles by ANOVA-based, modeldependent and -independent methods. Int. J. Pharm., v.209, p. 57-67, 2000.

Recebido para publicação em 21 de julho de 2005. Aceito para publicação em 28 de agosto de 2006. 